\title{
Local recommendations for death scene investigation of sudden unexpected death in infants (SUDI) based on prospective observations
}

T Bennett, BSc (Forensic Science and Genetics), BSc Hons (Forensic Genetics), MPhil (Biomedical Forensic Science); L J Martin, MB ChB, Dip For Med (SA), MMed (Forensic Path), FForPath (SA); L J Heathfield, BSc (Genetics and Physiology), BSc (Med) Hons (Human Genetics), MSc (Forensic Science) (UK), PgDip (Health Professions Education), PhD (Human Genetics)

Division of Forensic Medicine and Toxicology, Department of Pathology, Faculty of Health Sciences, University of Cape Town, South Africa

Corresponding author: L J Heathfield (laura.heathfield@uct.ac.za)

Background. The analyses of death scenes of sudden unexpected death in infants (SUDI) form an integral part of postmortem investigations. However, previous research has suggested that death scene investigation in SUDI cases is inconsistent and limited in South Africa. Objectives. To suggest realistic and feasible improvements for SUDI scene investigation by means of prospective observation. Methods. Ten SUDI cases were followed up from death scene until autopsy and detailed observations were made using a semi-structured checklist. Data were analysed in conjunction with published data from the same mortuary to suggest realistic improvements. Results. In all observed cases, the infant was moved prior to the arrival of forensic pathology officers; yet, reconstruction of the events leading to death were never demonstrated with a doll. The use of photography varied, with a median of 15 (standard deviation 6.5) photographs taken at each scene. However, critical photographs, such as those of medication, were often omitted. Furthermore, medicine was not collected from any scene. The use of documentation was inconsistent, where the intended longitudinal use was achieved in only 2 of 10 cases. Forms were inadequately filled in, due to the sensitivity or lack of understanding of various questions, rendering the forms incomplete.

Conclusions. Training of specialised staff should therefore focus on five areas: doll re-enactment, photography, handling of medicine, accurate use of relevant documentation and use of a glossary. The implementation of these recommendations is deemed to be feasible in a resource-scarce mortuary setting and could assist other mortuaries in the development of locally relevant strategies.

S Afr Med J 2021;111(5):448-452. https://doi.org/10.7196/SAMJ.2021.v111i5.15305

Death scene investigation (DSI) is an essential component of the investigation of sudden unexpected death in infants (SUDI). ${ }^{[1]}$ SUDI is the rapid and unanticipated death of an infant $<1$ year of age. ${ }^{[2]}$ Should the cause of death of a SUDI case remain undetermined following exhaustive postmortem investigations (including DSI), then sudden infant death syndrome (SIDS) is diagnosed by exclusion. ${ }^{[1]}$

DSI provides indispensable information that can contribute to determining an accurate cause of death, ${ }^{[3,4]}$ and can be used to inform ancillary investigations at autopsy. DSI has assisted in providing more accurate information regarding the causes and risk factors of SUDI. ${ }^{[5]}$ Global guidelines have been developed by the Centers for Disease Control and Prevention (CDC) for SUDI DSI, ${ }^{[6]}$ and a number of countries have modified and/or adopted these. ${ }^{[7]}$ Some forensic mortuaries follow national or regional protocols for the investigation of SUDI, ${ }^{[7-9]}$ whereas others, such as those in South Africa (SA), lack a uniform national protocol to investigate SUDI. ${ }^{[10]}$

In 2013, a multicentre study showed that SUDI DSI in SA is largely neglected. ${ }^{[10]}$ In 2016, a further SA study demonstrated that a standardised investigation for a cohort of 18 SUDI cases was possible. ${ }^{[5]}$ Another study at Salt River Mortuary, Cape Town, SA, one of the largest and busiest mortuaries in the country, found that only $59.2 \%$ of death scenes in SUDI cases were visited, and never when the body was admitted from a medical facility. No case showed fully completed paperwork. ${ }^{[1]}$ While this study identified many gaps in the DSI procedure, it was performed retrospectively and therefore did not have the insight as to whether the documentation actively reflected practices in these cases. Therefore, the aim was to prospectively observe the SUDI investigation process and use these data, together with past research ${ }^{[11]}$ to suggest realistic and feasible improvements for SUDI DSI in resource-constrained settings.

\section{Methods}

Salt River Mortuary was used as the study setting, where prospective information regarding the scope and consistency of current DSI practices for SUDI cases was gathered. Salt River Mortuary is an academic facility that investigates $\sim 4000$ deaths per year from the Western Metropole of the City of Cape Town. ${ }^{[12,13]}$

Between 21 July 2018 and 24 September 2018, 10 SUDI cases were included in this study, using convenience sampling. The forensic pathology officers were accompanied to the death scenes of SUDI cases and detailed observations were made using a semi-structured checklist (Supplementary Table 1: http://samj.org.za/public/sup/15305.pdf). This list was used to record the activities that took place at the scene, including time spent on the scene and evidence documented and/or collected by forensic pathology officers. No personal information was gathered at the scene with regard to the infants, families or personnel involved in the process.

Cases were longitudinally followed up throughout the process, which included transport of the body to the mortuary, family interview and legal identification process at the mortuary (typically a few days later), review of the clinical history and observation of the autopsy. The process was done to observe how the information gathered on the scene and the interview process influenced postmortem practices, and to gain insight regarding the type and extent of 
information documented. These visits were purely observational and no interventions took place.

The semi-structured checklist used in this study (Supplementary Table 1: http://samj.org.za/public/sup/15305.pdf) was independent from the official document (known as the FPS006(b)) used to investigate SUDI cases at Salt River Mortuary. Based on the international CDC guidelines, ${ }^{[14]}$ the FPS006(b) form consists of 7 sections $(A-G)$, where it is intended that the first 3 sections are completed contemporaneously on the scene and the remaining 4 are completed at the mortuary facility.

The information gathered was collated into a spreadsheet using Microsoft Excel 2013 (Microsoft Inc., USA), which, along with GraphPad Prism Version 6.1 (Prism, USA), was used to perform descriptive statistics and for visual representation of the data. The data were quantitative and categorical as far as possible, with the minority being qualitative.

\section{Ethical approval}

This study received ethical approval from the Human Research Ethics Committee, Faculty of Health Science, University of Cape Town (ref. no. HREC 218/2018). The forensic pathologists and forensic pathology officers were also informed of the study and given the opportunity to opt out if they did not wish to be observed.

\section{Results and discussion}

It was noticed that the staff at Salt River Mortuary operated within an exceptionally busy setting and gave their utmost towards the investigations into the multitude of SUDI cases admitted per month. During observations, compassion and respect were shown by the forensic pathology officers towards the families on the scenes and at the mortuary. When considering the SUDI investigation at Salt River Mortuary, many investigative procedures were in place and efforts towards international standards were noted. In particular, the implementation of the FPS006(b) document was aligned with global standards, and when present on the scene, the DSI appeared to be well conducted. Further, a comprehensive clinical history (via the 'Road to Health' documentation) was received in most cases, which was useful for forensic pathologists to consult during their investigations.

Moreover, 'Child death review' meetings were held monthly to discuss all child death cases and expedite legal and social decisionmaking. Child death review meetings encompass multidisciplinary committees that systematically review all child deaths and serve as a public health model of prevention of child fatalities. ${ }^{[15]}$ Therefore, externally, it appears that all criteria were in place to ensure the maximum effectiveness of SUDI investigations. However, a previous retrospective study showed that DSI of SUDI cases was not fully complete in a single case over a 2-year period. ${ }^{[11]}$ That study suggests that there was a misalignment between the procedures in place and implementation compliance.

Ten death scenes of SUDI cases were therefore prospectively observed to gather richer details pertaining to the longitudinal investigation to identify areas for improvement. Such insight is not possible through retrospective studies, as it is not clear whether the documentation correctly reflects what was done, or if the investigation was comprehensive and accurate. These prospective analyses of DSI practices, combined with retrospective data available from the same facility, ${ }^{[11]}$ allowed us to propose recommendations that we deem feasible and locally relevant. The recommendations presented below are integrated with the discussion.

\section{Recommendation 1: Re-enactment of the scene}

Forensic pathology officers attend death scenes to gather information that is beneficial for cause and circumstance of death determination. The investigations observed during the current study entailed documentation of the scene to gather such information, e.g. the position of the infant and household risk factors. However, in all 10 scenes, there were difficulties in the documentation of some aspects due to the infant being moved before the arrival of the forensic pathology officers. Therefore, the position in which the infant was found dead, description of the airway, wedging, co-sleeping, overlaying and whether the infant was tightly wrapped or swaddled could not be accurately documented. All these aspects are important for defining the cause of death as SIDS or another cause of death, e.g. suffocation. ${ }^{[4]}$

A retrospective analysis of SUDI cases in 2016 and 2017 in the same mortuary setting showed that in $66.9 \%(n=304)$ the infant had been moved prior to the arrival of the forensic pathology officers. In a further $13.4 \%$ of cases, it was unknown whether the infant had been moved. ${ }^{[16]}$ In this prospective study, as well as in the retrospective study, only a single re-enactment of the scene took place.

Scenarios in which the infant had been moved bring rise to issues regarding how the scene and its documentation should be handled. Sometimes the scene was documented as it was found and at other times the family was questioned in an attempt to reflect the scene at the time of death (i.e. before the infant was moved). To address this, a checkbox for whether the infant had been moved or not, should be included on the FPS006(b) form. Additionally, it ought to be noted whether documentation at the scene was influenced by questioning of the family.

Re-enactment of the scene is a peer-reviewed method, which allows the events leading up to death to be documented. The individual who found the baby is asked to depict how the infant was found, and this is photographed. ${ }^{[17]}$ It has been shown that scene investigations that include re-enactment aid cause of death determination by providing key information regarding risk and protective factors. ${ }^{[17-19]}$ CDC guidelines for the implementation of re-enactment are available and would require little adjustment to be introduced in our context. ${ }^{[20]}$ Re-enactments have been performed successfully with and without a doll, ${ }^{[18]}$ which indicate that even in our resource-scarce environment, dolls need not be purchased. Instead, other readily available objects could be substituted. Re-enactment, with subsequent photography, is recommended to be implemented at Salt River Mortuary, as this was not seen at any DSI.

\section{Recommendation 2: Photography and handling of medication}

Photography is unarguably one of the most important aspects of evidence gathering and is an effective tool for the retrospective explanation of a scene to people who were not present. ${ }^{[21]}$ Photographs were taken at all 10 scenes, with between 7 and 28 photographs per scene (median 15 (standard deviation (SD) 6.5)). These photographs were mostly of good quality, with only 2 of the total of 155 being blurred. There appears to have been a change in practice, as considerably fewer photographs were taken in 2016 and 2017 (median 3 (range 0 - 33)), with $5 \%$ being blurred. ${ }^{[16]}$

The CDC have established photography guidelines, not only for doll re-enactment, but for SUDI scenes in general. ${ }^{[20,22]}$ These are applicable in our setting and could be easily implemented with the use of a photography checklist to ensure that nothing is overlooked. This type of guided approach is envisaged to assist forensic pathology officers to determine the relevant aspects that should be 
photographed, which would provide the forensic pathologist with the necessary information to make informed decisions. ${ }^{[20]}$

\section{Recommendation 3: Physical and administrative handling of a scene with medication}

The abuse of illicit substances is a major global public health issue, and is rife in SA, making this evidence of great importance to all death investigations. ${ }^{[23]}$ The presence of illicit substances in an infant can have criminal and/or social implications, and could be indicative of neglect or abuse. ${ }^{[24,25]}$ Substance use by parents may lead to altered behaviour of caregivers, which could also result in an unsafe environment for the infant. ${ }^{[24,25]}$ Further, in SA, toxicological analysis is not done routinely, but is rather prompted by a specific case history and context. ${ }^{[26]}$ Evidence of the presence/use of drugs, cigarettes and/or alcohol in the household, is therefore essential to document.

From scene observations during this study and from retrospective data $^{[11]}$ it was apparent that the approach to finding medication, cigarettes and/or alcohol was grossly inconsistent. There was also no standardised approach to 'medical history-taking' and what was required when medicine was present on the scene. In some cases, only the national clinic card was consulted to document the history; sometimes, the caregiver was consulted regarding the history, and in other cases, both or none was done. Since the clinic card is brought to the mortuary a few days later, efforts on the scene should rather be geared towards asking the family for medical information, based on scene findings. A practical recommendation is the incorporation of questions regarding medical history into section $\mathrm{C}$ (scene investigation section) of the FPS006b document and stating on the form that the questions must be asked of the family and not collected from the clinic card.

It is mentioned in various protocols that all items that may have been administered to the infant (including medication), should be retained. ${ }^{[22,27,28]}$ Although these various protocols indicate that medication should be retained, it is recommended that local training and guidance pertaining to common medications must be implemented and prioritised for the forensic pathology officers. It should be standard practice that all medicine pertaining to the infant be collected (if not previously done by the SA Police Service), whereas medicine pertaining to the mother should be documented and photographed. Additionally, it is recommended that the FPS006(b) document should prompt the forensic pathology officer to perform these practices.

\section{Recommendation 4: Document completion and longitudinal use}

The use of the FPS006(b) form was longitudinally observed throughout the SUDI investigation. This documentation was correctly used from the death scene to the end of the autopsy in only 2 cases (Fig. 1). In the remainder, either new forms were used at the interview $(n=3)$ or the form was not present on the scene $(n=4)$. In one of the latter cases, the scene aspect was retrospectively completed after the scene visit, but before the interview stage. In only 9 of the 10 cases was the document passed on to the forensic pathologist for consultation (Fig. 1).

During the interview process at the mortuary, section D - G of the FPS006(b) form was sub-optimally completed; in no case was this form complete. These findings replicate those of the retrospective study, where no forms were complete (2016 and 2017). ${ }^{[11]}$ Therefore, while the appropriate forms were available at Salt River Mortuary, the correct completion of the documentation was problematic. The reasons for the apparent incomplete forms were probed during the observations, where during the family interview, it was often noted that if an answer to a question was 'no', it was merely left unmarked. Questions were also left unanswered when there was no corresponding option to circle. For example, the question: 'Was the following in the room where the baby slept to heat the room?', had the following answers to select: (i) electric heater; (ii) 'galley'; (iii) fire; or (iv) other; with no available option for 'none. This question was often unanswered, but it cannot be assumed that none of the heating appliances was present.

There were other, more sensitive, questions that were also frequently unanswered, including: 'Estimated monthly income'; 'Age of the mother'; and 'What schooling level did [the mother] achieve?' During observations, one of the male forensic pathology officers subsequently expressed his discomfort to the researchers, indicating that he felt it was not his place to ask a grieving and vulnerable woman such sensitive questions. He intentionally skipped more intimate questions, such as HIV status and whether contraception was used. Therefore, it is suggested that specially trained forensic pathology officers handle SUDI cases.

More pressing is the need for the paperwork to be used optimally and followed through accurately, and perhaps integrated within the overall electronic software utilised for casework management. For example, it could be compulsory to complete questions pertaining to the scene using electronic tablets, which are already available on scene, before completion of the scene can be logged. It would also be compulsory to answer all questions, and skipping questions would not be possible if one has to proceed to the next investigative phase. This would be a long-term project requiring resources to develop the software, to provide training for use of the software and to ensure maintenance of the electronic tablets. However, it would not only lead to completed documentation, but also assist with the chain of documentation, and limit the chances for forms to be misplaced.

\section{Recommendation 5: Localised revisions and incorporation of a glossary}

Death scene forms must be revised to be locally relevant, less ambiguous and must include a glossary. As mentioned above, the FPS006(b) document used in SUDI investigations by Salt River Mortuary is a proxy for the CDC's 'sudden, unexplained infant death reporting form' (SUIDRF). Efforts have already been made to adapt this form to an SA setting. However, there are aspects that are still irrelevant, e.g. checking whether 'electric baseboard heat' was present, as such details are almost non-existent in SA. Indeed, documentation of the use of a heater, or any other heat source, was rarely recorded in this study. However, checking for adequate ventilation is locally relevant, as many families live in informal dwellings, which are not adequately ventilated. To this end, terms such as 'informal housing' and 'adequate ventilation' also need to be defined within the local context, where many people live in dwellings with 'formal' bricked walls but 'informal' corrugated irons roofs. Defining these terms would lead to data being objective and meaningful.

To address this gap, the FPS006(b) form should include an appended glossary, providing definitions and descriptions for the categories that must be assigned. This is a relatively easy, once-off task, which could be performed by a task team, piloted and then reviewed to assess its impact. It may prove highly beneficial to ensure that the correct information is relayed and questioned.

The concept of a glossary could also be extended to the questionnaire session with the family, as English is not the first language of a number of forensic pathology officers; therefore, definitions should be included for words that appear on the documentation that are 


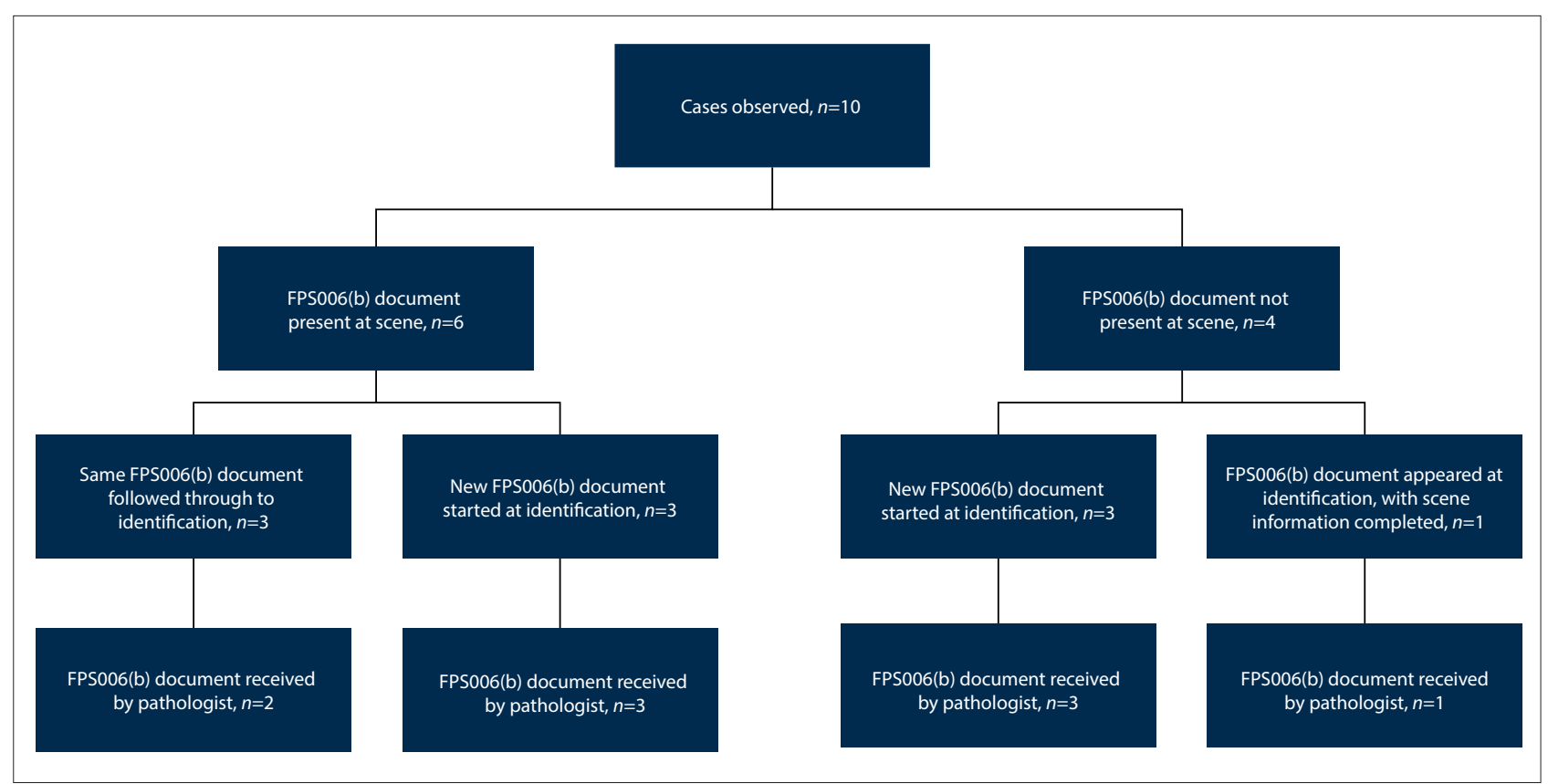

Fig. 1. Longitudinal use of FPSO06(b) documentation for cases of observed sudden unexpected death in infancy. In 2 of the 10 cases the FPSO06(b) document was used longitudinally throughout the entire process.

not well known or not frequently used. For example, words such as 'antenatal' and 'Kangaroo mother care' could not always be described by the forensic pathology officer to the family. Some questions also need to be worded in such a way as to reduce misinterpretation by non-native English speakers. Some words or phrases may be understood by the forensic pathology officer, who may struggle to explain these to the family. A glossary could serve as a reminder of the meanings of words or as a guideline for how things could be explained. There should also be a feedback loop, whereby forensic pathology officers can bring ambiguous questions to the attention of the Forensic Pathology Services authorities - then revised forms can be issued.

\section{Training}

An overarching recommendation to accompany all abovementioned recommendations is the need for training of forensic pathology officers in all aspects of SUDI investigation. Training is needed to guide the officers regarding the best practices with the available resources. Considering the finding of this study, training should first focus on three areas: (i) scene photography, to ensure that the photographs taken have evidential value; (ii) identification and handling of medication and drugs on SUDI scenes; and (iii) correct completion of forms.

Salt River Mortuary is an academic facility (as are many others around the world) and therefore short courses and ongoing professional development on the relevant topics could be developed by partnering universities. These additional learning opportunities could assist in the understanding of forensic evidence on the scene and how best to preserve this, and should constitute part of formalised training.

\section{Study limitations}

The prospective observation was performed on a small number of cases. While it is important to emphasise that these observations cannot be generalised, they did provide valuable insight into why there were many gaps, and into the feasibility of our recommendations. Where possible, the information gathered prospectively was assessed together with data collected for 454 cases analysed in the retrospective case review, which was conducted in the same setting. The insight and recommendations were fed back to the management of Salt River Mortuary for consideration or possible incorporation into training.

\section{Conclusions}

There is no protocol for the routine performance of DSI for SUDI cases in SA. The use of the FPS006(b) document does not solely constitute a standard operating procedure for how DSI should be conducted, but it does guide the investigation. Therefore, the aim of this research was to investigate the current DSI processes of SUDI cases at Salt River Mortuary and suggest realistic and feasible ways to improve this investigation. A prospective observation of DSI for 10 SUDI cases, using a semi-structured checklist, was performed. The observations showed that the SUDI scenes were assessed with inconsistent levels of documentation or photography, backed up by retrospective data obtained at the same settings. Therefore, datagathering methods available were not implemented to their full potential and all available information was not documented. In all 10 scene observations, the infant had been moved prior to DSI, yet no re-enactment of the scene was carried out. The forms used to document the death scene (FPS006(b)) were not optimally utilised, which calls for a revision of the forms, as well as training on how to complete them.

It is understandable, but not ideal, that international guidelines, such as those set by the CDC, cannot always be followed in resource-limited settings. However, the findings support the need for a standardised approach to DSI, coupled with specialised training for staff, which can be performed on a national level. Based on the available resources, this should focus on five areas: (i) implementation of doll re-enactment and contemporaneous documentation of the moved infant; (ii) establishment of guidelines pertaining to photography; (iii) handling of medicine; (iv) accurate use of relevant documentation; and $(v)$ implementation of a glossary. These recommendations will not only benefit and improve the DSI, but also the subsequent stages in the death investigation. 
Declaration. The research for this study was done in partial fulfilment of the requirements for TB's MPhil (Biomedical Forensic Science) degree at the University of Cape Town.

Acknowledgements. We thank the staff at Forensic Pathology Services, Salt River Mortuary, for their co-operation with regard to the study.

Author contributions. TB: methodology, investigation, formal analysis, writing of the original draft; LJM: supervision, writing of the article and editing; LJH: conceptualisation, methodology, formal analysis, validation, resources, writing of the article and editing, funding acquisition and supervision.

Funding. We thank the National Research Foundation (NRF) for student funding for the first author and the University of Cape Town for the Postgraduate Publication Incentive award.

Conflicts of interest. None.

1. Krous HF, Beckwith JB, Byard RW, et al. Sudden infant death syndrome and unclassified sudden infant deaths: A definitional and diagnostic approach. Pediatrics 2004;114(1):234-238. https://doi. org/10.1542/peds.114.1.234

2. Moon RY, Horne RSC, Hauck FR. Sudden infant death syndrome. Lancet 2007;370(9598):1578-1587. https://doi.org/10.1016/S0140-6736(07)61662-6

3. Matthews TJ, MacDorman MF, Thoma ME. Infant mortality statistics from the 2013 period linked birth/infant death data set. Natl Vital Stat Rep 2015;64(9):1-30.

4. Lambert EAB, Parks SE, Camperlengo L, et al. Death scene investigation and autopsy practices in sudden unexpected infant deaths. J Pediatr 2016;174:84-90. https://doi.org/10.1016/j.jpeds.2016.03.057

5. Dempers JJ, Coldrey J, Burger EH, et al. The institution of a standardised investigation protocol for 5empers J], Coldrey J, Burger EH, et al. The institution of a standardised investigation protocol for
sudden infant death in the eastern metropole, Cape Town, South Africa. J Forensic Sci 2016;61(6):1508-1514. sudden infant death in the eastern metropole,
https://doi.org/10.1111/1556-4029.13204

6. Centers for Disease Control and Prevention (CDC). Guidelines for death scene investigation of sudden, unexplained infant deaths: Recommendations of the interagency panel on sudden infant death yndrome. Morb Mortal Wkly Rep 1996;45:1-6.

7. Bennett T, Martin LJ, Heathfield LJ. Global trends in the extent of death scene investigation performed for sudden and unexpected death of infant (SUDI) cases: A systematic review. Forensic Sci Int 2019;301:435-444. https://doi.org/10.1016/j.forsciint.2019.06.013

8. Du Toit-Prinsloo L, Dempers JJ, Wadee S, et al. The medico-legal investigation of sudden, unexpected and/or unexplained infant deaths in South Africa: Where are we - and where are we going? Forensic Sci Med Pathol 2011;7(1):14-20. https://doi.org/10.1007/s12024-010-9184-7

9. Howatson AG. The autopsy for sudden unexpected death in infancy. Curr Diagn Pathol 2006;12:173-183. https://doi.org/10.1016/j.cdip.2006.03.008

10. Du Toit-Prinsloo L, Dempers JJ, Verster J, et al. Towards a standardised investigation protocol in sudden unexpected deaths in infancy in South Africa: A multicentre study of medio-legal investigation sudden unexpected deaths in infancy in South Africa: A multicentre study of medio-legal investigation
procedures and outcomes. Forensic Sci Med Pathol 2013;9(3):344-350. https://doi.org/10.1007/ procedures and outco
s12024-013-9427-5
11. Bennett T, Martin LJ, Heathfield LJ. A retrospective study of death scene investigation practices for sudden unexpected death in infants (SUDI) in Cape Town, South Africa. Forensic Sci Med Path sudden unexpected death in infants (SUDI) in Cape Town,

12. Western Cape Government Department of Health. Forensic Pathology Services: Our facilities. https://www.westerncape.gov.za/general-publication/our-facilities (accessed 12 June 2018).

13. Clark C, Mole CG, Heyns M. Patterns of blunt force homicide in the West Metropole of the City of Cape Town, South Africa. S Afr J Sci 2016;113(5):1-6. https://doi.org/10.17159/ sajs.2017/20160214

14. Centers for Disease Control and Prevention (CDC). Sudden infant death syndrome - United States, 1983 - 1994. Morb Mortal Wkly Rep 1996;45:859-863.

15. Christian CW, Sege RD. Policy statement - child death review. Am Acad Pediatr 2010;126(3):592-596. https://doi.org/10.1542/peds.2010-2006

16. Bennett $T$. Exploring the medico-legal death scene investigation of sudden unexpected death of Bennett T. Exploring the medico-legal death scene investigation of sudden unexpected death of
infants at Salt River Mortuary, Cape Town, South Africa. MPhil thesis. Cape Town: University of infants at Salt Rive Town, 2018 .

17. Kemp JS, Kowalski RM, Burch PM, et al. Unintentional suffocation by rebreathing: A death scene and physiologic investigation of a possible cause of sudden infant death. J Pediatr 1993;122(6):874-880. https://doi.org/10.1016/s0022-3476(09)90010-5

18. Sauber-Schatz EK, Sappenfield WM, Shapiro-Mendoza CK. Comprehensive review of sleep-related sudden unexpected infant deaths and their investigations: Florida 2008. Matern Child Health 2015;19(2):381-390. https://doi.org/10.1007/s10995-014-1520-1

19. Byard RW, Beal SM. V-shaped pillows and unsafe infant sleeping. J Paediatr Child Health 1997;33(2):171-173. https://doi.org/10.1111/j.1440-1754.1997.tb01024.x

20. Diebold K. Conducting the doll reenactment: Concurrent and retrosepctive scene strategies. In Centers for Disease Control and Prevention. Sudden, Unexplained Infant Death Investigation: Guidelines for the Scene Investigator. Georgia: CDC, 2007:170-18

21. Robinson EM. Crime Scene Photography. USA: Elsevier, 2016:14.

22. Ernst MF, Nunez S, Jentzen J, et al. Conducting scene investigations: Residence - incident scene death scene. In: Centers for Disease Control and Prevention. Sudden, Unexplained Infant Death Investigation: Guidelines for the Scene Investigator. Georgia: CDC, 2007:140-169.

23. Auckloo M, Davies B. Post-mortem toxicology in violent fatalities in Cape Town, South Africa: A preliminary investigation. J Forensic Leg Med 2019;63:18-25. https://doi.org/10.1016/.j.jlm.2019.02.005

24. Osler T, Baker SP, Long W. A modification of the injury severity score that both improves accuracy and simplifies scoring. J Trauma 1997;43(6):922-926.

25. Byard RW, Shipstone RA, Young J. Continuing major inconsistencies in the classification of unexpected infant deaths. J Forensic Leg Med 2019:64:20-22. https://doi.org/10.1016/j jflm.2019.03.007

26. Louw A. Post-mortem toxicological investigations in a paediatric population. MPhil thesis. Cape Town: University of Cape Town, 2019

27. Johnson C, Sumner C, Rebello A. Merseyside joint agency protocol: Sudden unexpected death in infancy (SUDI) for babies/children from 0 up to 2 years. 2012. http://liverpoolscb.proceduresonline. com/pdfs/sudi.pdf (accessed 20 March 2018).

28. Association of Chief Police Officers. A guide to investigating child deaths. 2014. https:/ lincolnshirescb.proceduresonline.com/pdfs/acpo_invest_child_deaths.pdf (accessed 18 March 2021)

Accepted 5 November 2020 